\title{
EL TAMAÑO, PERO NO LA FORMA, REFLEJARÍA DIFERENCIAS EN LAS MANDÍBULAS DE DOS ESPECIES DE MURCIÉLAGOS DE HOJA NASAL (PHYLLOSTOMUS LACÉPÈDE, 1799) (CHIROPTERA, PHYLLOSTOMIDAE)
}

\author{
P. M. Parés-Casanova \\ Department de Ciència Animal, Universitat de Lleida, Av. Rovira Roure, 191, 25198 Lleida, Cataluña, España \\ E-mail: peremiquelp@ca.udl.cat. ORCID iD: http://orcid.org/0000-0003-1440-6418
}

\section{RESUMEN}

La mandíbula es un modelo para la evolución de las estructuras morfológicas complejas debido a que presenta una integración del desarrollo de sus diferentes estructuras. A diferencia de otras partes del cráneo, que albergan estructuras con funciones diferentes (protección del cerebro y de los órganos sensoriales), la principal demanda funcional para la mandíbula se relaciona con la alimentación. Por ello, es esperable que sea la dieta su principal agente selectivo. En esta investigación se presenta un estudio basado en morfometría geométrica, de mandíbulas pertenecientes a dos especies de murciélagos de hoja nasal, Phyllostomidae: el murciélago nariz de hoja pálido (Phyllostomus discolor Wagner, 1843) $(n=29)$ y el murciélago nariz de hoja lanceolada [Phyllostomus hastatus (Pallas, 1767)] $(n=6)$, ambas especies omnívoras, con tendencia nectarívora. Las dife-rencias en tamaño y forma de la mandíbula fueron examinadas usando 13 hitos anatómicos situados en la cara lateral de las hemimandíbulas. Los resultados revelaron diferencias entre ambas especies para el tamaño, pero no para la forma, siendo insignificante la variación alométrica. Se concluye que una alimentación parecida para ambas especies ha hecho evolucionar hacia una forma mandibular similar, aunque el tamaño para ambas haya evolucionado de manera independientemente.

Palabras clave: alometría; Phyllostomidae; Phyllostomus discolor; Phyllostomus hastatus.

\section{ABSTRACT}

Size, but not shape, would reflect mandibular differences in two spear-nosed bats (Phyllostomus Lacépède, 1799) (Chiroptera, Phyllostomidae)

The mandible is a model for the evolution of complex morphological structures because it presents a developmental integration of its different parts. Unlike other parts of the skull that harbour different functions (protect-ing the brain and sensory organs), the mandible's main functional demands are related to feeding, and dietary changes are expected to be the main selective agent for its structure. Here it is presented a geometric morpho-metric study of mandibles belonging to two species of New World leaf-nosed bats, Phyllostomidae: Pale Spear-nosed Bat (Phyllostomus discolor Wagner, 1843) $(n=29)$ and Greater Spear-nosed Bat [Phyllostomus hastatus (Pallas, 1767)] $(n=6)$, both of them with an omnivorous with a nectarivorous shift feeding habit. Differences in size and shape of mandible were examined using 13 landmarks plotted on lateral aspect. The results revealed species differences for size but not for shape, being allometry an unimportant component of interspecific varia-tion. It is concluded that similar feeding specialization in both species would have evolved towards similar man-dibular shape, with size having evolved quite independently in both species.

Key words: allometry; Phyllostomidae; Phyllostomus discolor; Phyllostomus hastatus. mandibulas de dos especies de murciélagos de hoja nasal (Phyllostomus Lacépède, 1799) (Chiroptera, Phyllostomidae). Grael/sia, 74(2): e081. https://doi.org/10.3989/graellsia.2018.v74.211 


\section{Introducción}

Existen buenas razones teóricas para esperar que animales que difieran en tamaño difieran también en forma, como respuesta al principio simple que predice cambios en las proporciones como resultado de cambios en el tamaño (Pélabon et al., 2014). Se han publicado, en efecto, numerosísimos estudios que demuestran los cambios en la forma de los organismos según el tamaño. En el caso de los mamíferos de Colombia, y para el caso concreto de los quirópteros, se han encontrado diferencias de tamaño en varias especies (Rodríguez-Posada \& Sánchez-Palomino, 2009), incluyendo las de la familia Phyllostomidae existentes en este país (Mantilla-Meluk et al., 2009).

Los filostómidos, los murciélagos de hoja nasal, se encuentran distribuidos a lo largo de América Central y del Sur, desde México hasta el norte de Argentina, habiendo experimentado una radiación adaptativa sin precedentes en cuanto a su diversidad ecológica y morfológica (Monteiro \& Nogueira, 2011). Las adaptaciones que presentan los filostómidos son, efectivamente, de las más variadas y diversas dentro del orden Chiroptera, siendo la especialización trófica la clave en su evolución (Monteiro \& Nogueira, 2011). El género Phyllostomus Lacépède, 1799, que da nombre a la familia, está representado por cuatro especies de murciélagos de mediano a gran tamaño, todas ellas registradas en Colombia: $P$. discolor Wagner, 1843, P. elongatus (É. Geoffroy, 1810), P. hastatus (Pallas, 1767) y P. latifolius (Thomas, 1901) (Mantilla-Meluk et al., 2009; Nogueira et al., 2009; Rodríguez-Posada \& Sánchez-Palomino, 2009).

El murciélago nariz de hoja pálido (Phyllostomus discolor) oscila entre 89 y $109 \mathrm{~mm}$ de longitud total y se extiende desde Oaxaca y Veracruz (México) a Guayanas, sudeste de Brasil, Bolivia, Paraguay, Perú y Venezuela (Kwiecinski, 2006). El murciélago nariz de hoja lanceolada (Phyllostomus hastatus) es claramente mayor, con una longitud total de aproximadamente 124-131 mm, y se encuentra ampliamente distribuido, pudiéndose encontrar desde Honduras a Paraguay y sudeste de Brasil a Trinidad.

La diversidad trófica de los murciélagos se refleja en la diversidad estructural de su aparato masticatorio (Freeman, 1998; Dumont \& Nicolay, 2006). Si las principales demandas funcionales de la mandíbula se relacionan con la alimentación, parecería que la dieta sería su principal agente selectivo (Dumont \& Nicolay, 2006). Phyllostomus discolor está descrita como una especie omnívora, con tendencia nectarívora (Kwiecinski, 2006), lo mismo que P. hastatus (Santos et al., 2003) y es este régimen alimentario el que explica la singular elongación del hocico en este género (Monteiro \& Nogueira, 2011). Centramos pues este estudio en la variación del contorno de la mandíbula (tamaño y forma) en estas dos especies de Phyllostomus, de tamaño dispar pero régimen alimenticio similar, para saber si sus igualdades dietéticas han modelado formas mandibulares distintas o no. O dicho de otro modo, la similitud de dietas entre $P$. hastatus y $P$. discolor se refleja en una igualdad de la forma de la mandíbula?

\section{Material y métodos}

Recolección de datos. Se analizaron 35 mandíbulas pertenecientes a dos especies de Phyllostomus: P. discolor $\left(\mathrm{n}=29: 9 \hat{\jmath} \hat{\sigma}^{2}, 6\right.$ 우 y 14 de sexo descono-

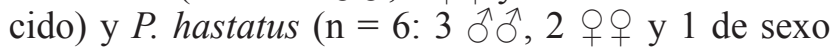
desconocido) depositados en la colección del Instituto de Ciencias Naturales de la Universidad Nacional de Colombia. Cada espécimen había sido identificado taxonómicamente hasta el nivel de especie y había sido recogido para otros estudios, no llevándose pues a cabo ninguna captura ni sacrificio ex professo de animales para esta investigación. Los especímenes con puntos faltantes por un mal estado de conservación o por no ser estos puntos poco claros fueron omitidos.

Obtención de puntos de referencia. Las fotografías digitales de las hemimandíbulas izquierda, por su cara lateral, se obtuvieron con una cámara digital Nikon D1500 equipada con un teleobjetivo Nikon DX de 18-105 mm. En cada imagen fue incluido un patrón lineal de referencia. Las imágenes se guardaron en formato jpg. Un total de 13 puntos de interés fue digitalizado utilizando el software de versión 2.21 TpsDig (Rohlf, 2010) (Fig. 1). Estos puntos de referencia elegidos, similares a los utilizados por otros autores en quirópteros (Mancina \& Balseiro, 2010), estaban presentes en todas las muestras, y teniendo en cuenta que se reparten por toda la morfología de la cara lateral del hemimandíbula, se consideró que engloban todas las unidades morfogenéticas mandibulares -alvéolos, apófisis y puntos de máxima curvatura-.

Para todas las muestras se obtuvo pues una serie de coordenadas cartesianas 2D $(x, y)$ de cada uno de los 13 hitos estudiados.

Superposición de Procrustes. La forma fue obtenida de las coordenadas cartesianas originales estandardizando el tamaño de cada espécimen y reduciendo al mínimo las diferencias translacionales y posicionales mediante un análisis generalizado de Procrustes (Gower, 1975). Muy sucintamente, el método consiste en ajustar cada espécimen a fin que la distancia entre cada punto cartesiano y el centroide sea el mínimo (Webster \& Sheets, 2010).

Análisis estadístico. Para obtener información sobre la forma, los datos fueron primero superpuestos mediante el paquete IMP CoordGen8 (Sheets, 1998). Utilizamos el tamaño de centroide (raíz cuadrada de las distancias al cuadrado sumadas de cada punto de referencia al centroide de la configuración) (Swiderski, 


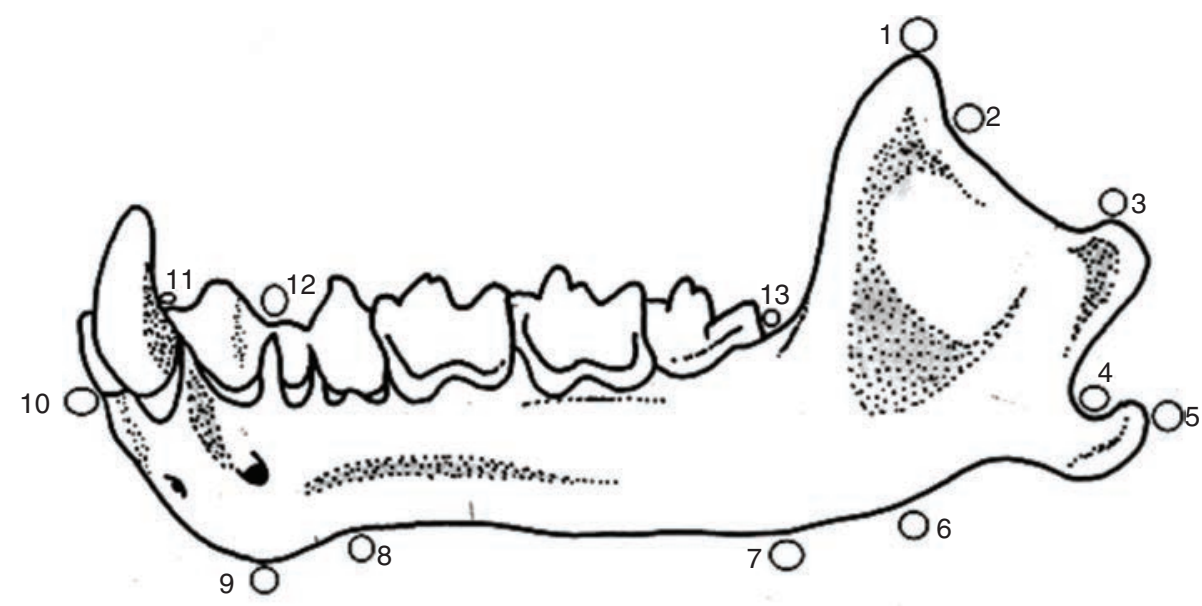

Fig. 1.- Mandíbula de Phyllostomus sp. mostrando los 13 hitos utilizados para capturar la forma y el tamaño.

Fig. 1.- Phyllostomus sp. mandible showing 13 landmarks used to capture form.

1 Punta del processus coronoides.

2 Punto de máxima concavidad de la incisura mandibulae.

3 Punto más dorsal del caput mandibulae.

4 Punto de máxima concavidad entre el punto más dorsal de la superficie condilar y el punto más caudal del proceso post-condilar.

5 Punto más caudal del proceso post-condilar.

6 Punto más ventral en la proyección perpendicular del extremo del proceso coronoide.

7 Punto más ventral en la proyección perpendicular del margen alveolar de la serie molar.

8 Proyección ventral perpendicular al punto más caudal del margen alveolar de la pM.

9 Proyección ventral perpendicular al punto más rostral del margen alveolar de la $\mathrm{pM}_{1}$.

10 Punto más rostral de la mandíbula.

11 Punto más rostral del margen alveolar de $\mathrm{pM}_{1}$.

12 Punto más caudal del margen alveolar de $\mathrm{pM}_{1}$.

13 Punto más caudal de margen alveolar de la serie molar.

2003) como una medida geométrica del tamaño de la mandíbula. Los vectores de lollipop, que fueron utilizados para presentar gráficamente el cambio medio en la dirección y significa la magnitud del desplazamiento del punto de referencia, se obtuvieron con el software MorphoJ, versión 1.06c (Klingenberg, 2011).

Se utilizó el paquete TpsSmall, versión 1.29 (Rohlf, 2015) para evaluar la correlación entre las distancias 2D y las distancias euclidianas en el espacio tangente de la proyectada. La correlación obtenida fue muy lineal para todos los datos $(r=1.000$; pendiente $b=0.9993$ ), lo que sugiere que el espacio tangente es una aproximación adecuada a Kendall y que ningún espécimen desviado sensiblemente desde la línea de regresión lineal (Webster \& Sheets, 2010). Así, aunque la mandíbula no es un objeto perfectamente coplanar, el autor considera que el enfoque bidimensional para su estudio ha implicado una pérdida limitada de información. Se analizó la posible diferencia de forma entre sexos por medio de un análisis multivariado de varianza NPMANOVA (Non-Parametric-MultivariateANalysis-Of-VAriance). Como no aparecieron diferencias de forma entre machos y hembras $(P$. discolor:

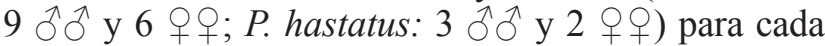
una de las especies estudiadas $(p>0.810)$ todos los ejemplares se agruparon para cada especie independientemente del sexo.
Análisis alométrico. La alometría estática denota cambios en la forma relacionada con el tamaño medidos en individuos diferentes en la misma etapa de desarrollo dentro de una población o especie (Pélabon et al., 2014; Klingenberg, 2016). Para evaluar este efecto se exploraron los cambios en la forma versus el tamaño de centroide (datos logarítmicamente transformados) mediante una regresión multivariante. El paquete TpsRegr, versión 1.36 (Rohlf, 2015) fue utilizado para obtener esta regresión.

Comparación del tamaño y la forma. El tamaño de la mandíbula se comparó entre especies con la prueba $U$ de Mann-Whitney, y el de la forma, con un test NPMANOVA con distancia de Mahalanobis. Para este análisis estadístico se recurrió al paquete estadístico PAST, versión 2.17c (Hammer et al., 2001). El nivel de error tomado en cuenta para todas las pruebas estadísticas fue $\alpha=0.05$.

\section{Resultados}

Los valores promedios del tamaño de centroide fueron 360.23 y 502.21 para $P$. discolor y $P$. hastatus, respectivamente, reflejando la prueba de MannWhitney diferencias estadísticas en ambas especies $(U=4, p<<0.001)$ (Fig. 2). Pero no aparecieron 


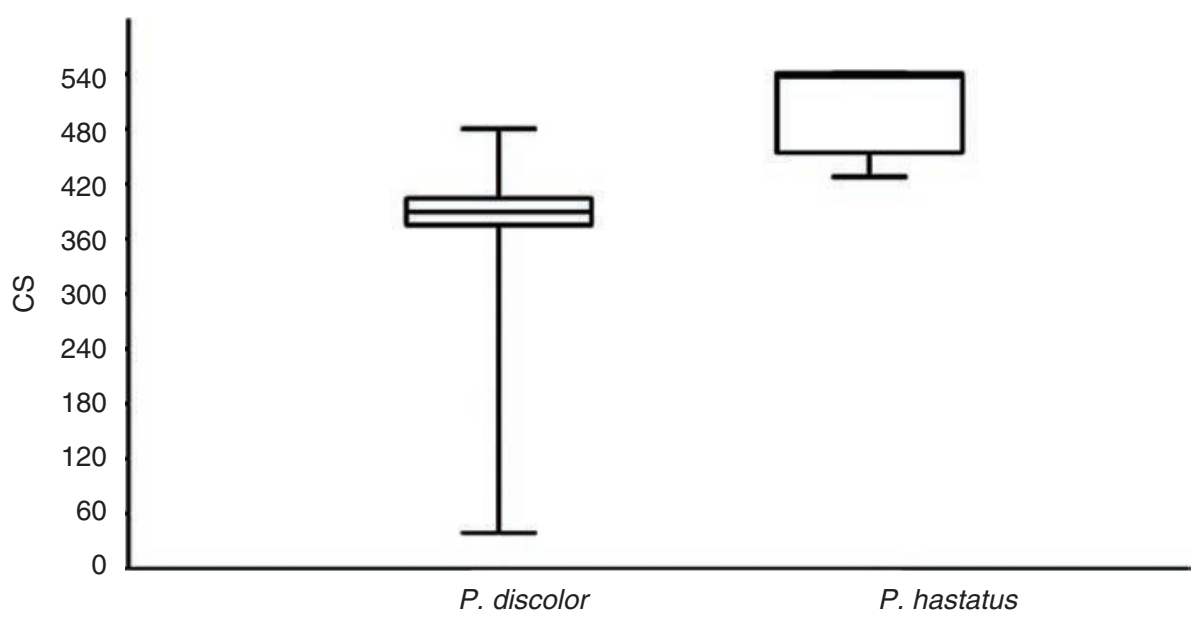

Fig. 2.- Comparación de tamaños (expresados como tamaño del centroide, CS) entre Phyllostomus discolor $(\mathrm{n}=29)$ y $P$. hastatus $(n=6)$. La mediana se muestra con una línea horizontal dentro de la caja. Las líneas horizontales cortas en la parte superior/inferior de la caja se sitúan a más/menos de 1,5 veces la altura de la caja de la caja. Los promedios del tamaño de centroide fueron de 360,23 y 502,21 para $P$. discolor y $P$. hastatus, respectivamente. El test de Mann-Whitney reflejó diferencias estadísticamente significativas entre ambas especies $(U=4, p<<0.001)$.

Fig. 2.- Comparison of sizes (expressed as Centroid Size, CS) between Phyllostomus discolor $(\mathrm{n}=29)$ and $P$. hastatus $(\mathrm{n}=6)$. The median is shown with a horizontal line inside the box. The means of CS were 360.23 and 502.21 for $P$. discolor and $P$. hastatus, respectively. Mann-Whitney test reflected statistical differences between the two $(U=4, p<<0.001)$.

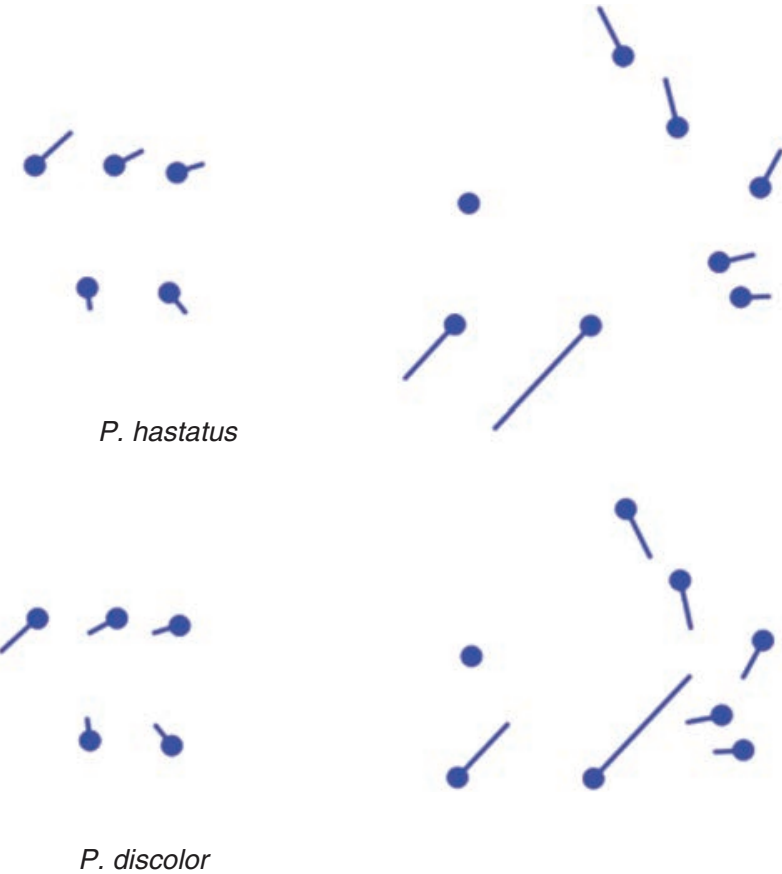

Fig. 3.- Figuras lollipop para Phyllostomus discolor $(\mathrm{n}=29)$ y $P$. hastatus $(n=6)$, que resumen gráficamente el cambio promedio en la dirección y la magnitud del desplazamiento de cada punto de referencia, para ambas especies. El cambio en la forma mandibular se asocia con estructuras mandibulares como cóndilo, coronoides y angular y la parte mental, pero no con la posición relativa y tamaño de las filas de los dientes.

Fig. 3.- Lollipop plots for Phyllostomus discolor $(n=29)$ and $P$. hastatus $(\mathrm{n}=6)$. They graphically summarize the mean change in direction and mean magnitude of landmark displacement for both species. Mandibular shape is associated with structures such as condylar, coronoid and angular processes, and mental part, but not with the relative position and sizes of tooth rows. diferencias en la forma, según reflejó el NPMANOVA $(\mathrm{F}=0.007, p=0,556)$ (Fig. 3). Los resultados de la regresión multivariante revelaron que la variación en la forma apenas se puede explicar por alometría (test generalizado de Goodall: $\mathrm{F}=0.502$, grados de libertad $=26,858, p=0.982$ ), es decir, que el tamaño predice muy poco cambios en la forma.

Así pues, el tamaño mandibular, pero no la forma, se mostró estadísticamente diferente entre $P$. discolor y $P$. hastatus. Dicho de otro modo, las mandíbulas están estructuradas del mismo modo entre las dos especies estudiada, quizás porque la mecánica de la mordida sería similar en $P$. discolor y $P$. hastatus. Por otra parte, la alometría mandibular no apareció como un componente importante de la diferenciación entre ambas especies.

\section{Discusión}

La morfometría se define como la descripción cuantitativa, el análisis y la interpretación del contorno (tamaño y forma) (Adams et al., 2013). Es un área fundamental de la investigación biológica, puesto que puede contribuir a la resolución de problemas taxonómicos y funcionales.

La especialización en la dieta crea demandas funcionales singulares, lo que a su vez determina cambios en la biomecánica mandibular, o sea que el comportamiento alimentario es relevante para entender la forma mandibular. En los filostómidos, está demostrado que existen estos cambios mandibulares relacionados con la fuerza de la mordedura, como son el tamaño relativo de los procesos mandibulares (coronoide y angular), la posición del arco cigomático y robustez, posición 
relativa y tamaño de las hileras dentales, todos ellos con independencia del tamaño (Nogueira et al., 2009). Además, aunque la dimorfía sexual de la mandíbula podría representar una forma de reducir la competencia entre los sexos, ya que permitiría a las hembras y los machos explotasen diferentes recursos tróficos, este dimorfismo no se ha detectado en las dos especies por nosotros estudiadas. De todos modos, en la bibliografía hay casos en que se encuentra dimorfismo sexual en la forma craneal (Power \& Tamsitt, 1973; Willig, 1983; Willig \& Hollander, 1995; RodríguezPosada \& Sánchez-Palomino, 2009). La interpretación de los resultados de los análisis de variación sexual intraespecífica queda de todos modos sujeta a comprobaciones con una muestra mayor y utilizando puntos anatómicos homólogos entre estudios.

En este estudio no se ha detectado tampoco ninguna diferencia en la forma mandibular entre las especies estudiadas, $P$. discolor y $P$. hastatus. Se explicaría ello porque los valores más altos en un rasgo se compensan con valores más bajos en otro, de lo que resulta un equilibrio general en la forma, equilibrio que a la vez se justificaría por un uso alimentario no diferencial. Dicho de otro modo: la presión selectiva trófica actuaría de manera uniforme en ambas especies.

En resumen: nos enfrentamos pues a una especialización de alimentación similar en Phyllostomus discolor y Phyllostomus hastatus, que requiere arquitectura funcional mandibular similar, aunque el tamaño haya evolucionado de forma independiente en ambas especies.

Obviamente, para corroborar nuestras conclusiones serían interesantes estudios con más cantidad de ejemplares y con nuevas técnicas, como la densitometría (aplicadas por Dumont \& Nicolay, 2006). Así mismo, deberíamos ser conscientes que en este estudio hemos recurrido a la morfometría geométrica, que analiza distancias entre puntos anatómicos discretos (Richtsmeier et al., 2002), por lo que la información obtenida ha sido en base a unas distancias determinadas, un subconjunto parcial de las posibles distancias entre estos puntos, por lo que podrían haber muchas medidas redundante, mientras que otras medidas quedarían submuestreadas o totalmente omitidas (Swiderski, 2003). Por consiguiente, la posible redundancia de las medidas contempladas y la omisión de los datos de forma independiente en diferentes regiones pueden haber introducido pequeñas diferencias en lo que atañe a comparación con el trabajo de otros autores referenciados, muchos de los cuales, por otro lado, han trabajado con morfometría lineal. De todos modos, puesto que no se detecta alometría en el caso estudiado, tampoco no se consideraría que la posible existencia de variables dependientes entre ellas haya podido representar una proporción exagerada de variación sesgada en la forma.

El presente trabajo es un primer paso para el estudio de las diferencias en la forma mandibular de estas dos especies de filostómidos, Phyllostomus discolor y Phyllostomus hastatus, y su relación con la dieta, pero la inclusión de semilandmarks para cuantificar curvas y superficies sería de gran valor para futuros trabajos sobre la estructura estudiada.

\section{Agradecimientos}

Agradecemos a Catalina Cárdenas y Hugo Fernando López por facilitarnos el acceso a las colecciones del Instituto de Ciencias Naturales de la Universidad Nacional de Colombia, en Bogotá, Colombia. También agradecemos a los revisores anónimos por sus comentarios y sugerencias. No se recibió ninguna ayuda financiera para esta investigación.

\section{Referencias}

Adams, D. C., Rohlf, F. J. \& Slice, D. E., 2013. A field comes of age: geometric morphometrics in the $21 \mathrm{st}$ century. Hystrix, 24(1): 7-14. https://doi.org/10.4404/ hystrix-24.1-6283

Dumont, E. R. \& Nicolay, C. W., 2006. Cross-sectional geometry of the dentary in bats. Zoology, 109(1): 66-74. https://doi.org/10.1016/j.zool.2005.08.004

Freeman, P. W., 1998. Form, function, and evolution in skulls and teeth of bats. Papers in Natural Resources, 9: 140-156.

Gower, J. C., 1975. Generalized procrustes analysis. Psychometrika, 40(1): 33-51.

Hammer, Ø., Harper, D. A. T. \& Ryan, P. D., 2001. PAST v. 2.17c. Palaeontologia Electronica, 4(1); 1-229.

Klingenberg, C. P., 2011. MorphoJ: An integrated software package for geometric morphometrics. Molecular Ecology Resources, 11(2): 353-357. https://doi.org/ 10.1111/j.1755-0998.2010.02924.x

Klingenberg, C. P., 2016. Size, shape, and form: concepts of allometry in geometric morphometrics. Development Genes and Evolution, 226(3): 113-137. https://doi.org/ 10.1007/s00427-016-0539-2

Kwiecinski, G. G., 2006. Phyllostomus discolor. Mammalian Species, 801: 1-11. https://doi.org/10.1644/801.1

Mancina, C. A. \& Balseiro, F., 2010. Variación en la forma de la mandíbula en Monophyllus redmani (Chiroptera: Phyllostomidae): análisis geométrico de la variación sexual y geográfica. Mastozoología Neotropical, 17(1): 87-95.

Mantilla-Meluk, H., Jimenez-Ortega, A. M. \& Baker, R., 2009. Phyllostomid bats of Colombia: annotated checklist, distribution, and biogegography. Special Publications, Museum of Texas Tech University, 56: 1-37.

Monteiro, L. R. \& Nogueira, M. R., 2011. Evolutionary patterns and processes in the radiation of phyllostomid bats. BMC Evolutionary Biology, 11: 137. https://doi.org/ 10.1186/1471-2148-11-137

Nogueira, M. R., Peracchi, A. L. \& Monteiro, L. R., 2009. Morphological correlates of bite force and diet in the skull and mandible of phyllostomid bats. Functional Ecology, 23(4): 715-723. https://doi.org/10.1111/j.1365-2435. 2009.01549.x

Pélabon, C., Firmat, C., Bolstad, G. H., Voje, K. L. \& Houle, D., 2014. Evolution of morphological allometry. 
Annals of the New York Academy of Sciences, 1320: 58-75. https://doi.org/10.1111/nyas. 12470

Power, D. M. \& Tamsitt, J. R., 1973. Variation in Phyllostomus discolor (Chiroptera: Phyllostomatidae). Canadian Journal of Zoology, 51(4): 461-468. https://doi. org/10.1139/z73-069

Richtsmeier, J. T., DeLeon, V. B. \& Lele, S. R., 2002. The promise of geometric morphometrics. Yearbook of Physical Anthropology, 45: 63-91. https://doi.org/10.1002/ ajpa. 10174

Rodríguez-Posada, M. E. \& Sánchez-Palomino, P., 2009. Taxonomía del género Phyllostomus (Chiroptera: Phyllostomidae) en Colombia. Mastozoologia Neotropical, 16(1): 153-168. Disponible en: http://www.scielo.org.ar/scielo. php?script $=$ sci_arttext\&pid=S0327-93832009000100013 [consultado, 22.10.2018].

Rohlf, F. J., 2010. Digitalized Landmarks and Outlines. Stony Brook. New York. Disponible en http://life. bio.sunysb.edu/morph/soft-dataacq.html [consultado, 22.10.2018].

Rohlf, F. J., 2015. The tps series of software. Hystrix, 26(1): 9-12. https://doi.org/10.4404/hystrix-26.1-11264
Santos, M., Aguirre, L. F., Vázquez, L. B. \& Ortega, J., 2003. Phyllostomus hastatus. Mammalian Species, 722: 1-6. https://doi.org/10.1644/0.722.1

Sheets, H. D., 1998. IMP: CoordGen8-Coordinate Generation Utility. Canisius College. Buffalo. Disponible en http://www3.canisius.edu/ sheets/CoordGenManual.htm [consultado, 22.10.2018].

Swiderski, D. L., 2003. Separating size from allometry: analysis of lower jaw morphology in the fox squirrel, Sciurus niger. Journal of Mammalian Evolution, 84(3): 861-876. https://doi.org/10.1644/BRB-025

Webster, M. \& Sheets, H. D., 2010. A practical introduction to landmark-based geometric morphometrics. The Palaeontological Society Papers, 16: 163-188.

Willig, M. R., 1983. Composition, microgeographic variation, and sexual dimorphism in Caatingas and Cerrado bat communities from northeastern Brazil. Bulletin of Carnegie Museum of Natural History, 23: 1-131.

Willig, M. R. \& Hollander, R. R., 1995. Secondary sexual dimorphism and phylogenetic constraints in bats: a multivariate approach. Journal of Mammology, 76(4): 981-992. https://doi.org/10.2307/1382592 